\title{
Modeling and Analysis of 802.11p Physical Layer for V2X Connected Transport Systems Considering Harsh Operating Conditions and HW Device Performance
}

\author{
Sergio Saponara, Alessio Gagliardi \\ *Dipartimento di Ingegneria della Informazione, Università di Pisa, via G. Caruso 16, 56122, Pisa, Italia
}

\begin{abstract}
Intelligent driving is a promising area for increased safety and comfort. Vehicular communication is an essential part to build such systems. This paper describes the modelling and the implementation of the IEEE 802.11p Physical (PHY) Layer to determine its reliability for vehicleto-everything (V2X), and particularly vehicle-to-vehicle (V2V), communications in the automotive field. A Matlab/ Simulink simulation is carried out to analyze not only the baseband processing of the transceiver, but also the RF hardware part, the physical channel in different operating conditions and environments, and all the main impairments and sources of interferences/noise. The transceiver model consists of three parts, the transmitter, the receiver and the intermediate channel block. The model can be used to explore the performance (bit-rate, successfully delivered packet-rate, latency,..) of $\mathrm{V} 2 \mathrm{X}$ links in different conditions (line-of-sight, non-line-of-sight), and environments (urban, suburban, rural and highway), considering single-hop or multi-hop networking, and allowing also dynamically changing the channel characteristics, or even using different modulation and coding schemes and physical transmission parameters. To assess the proposed V2X simulation tool, the simulation results are compared to the theoretical performance and to experimental results, obtained using the NEC LinkBird-MX C2X device. The proposed simulation tool can be useful to study the impact of vehicles distance, speed and operating scenario on the reliability of the communication system, once fixed the hardware apparatus, or to specify the performance of the hardware components needed to ensure a given $\mathrm{V} 2 \mathrm{X}$ communication performance.

Keywords: IEEE 802.11p Physical (PHY) Layer; Vehicle-toEverything (V2X); Vehicle-to-Vehicle (V2V); Wireless links
\end{abstract}

\section{Introduction}

The use of vehicle-to-vehicle (V2V) communication is an integral part of intelligent transportation systems (ITSs) [1-3], and will be a core technology for high (L4, L5) autonomous driving levels. Since vehicles are becoming increasingly intelligent, they will be able to detect potential dangers, such crash risk, unsafe overtake, obstacles, improving collision avoidance and reducing fatalities. A new need for $\mathrm{V} 2 \mathrm{X}$ sharing information is emerging. V2x communication, which involves vehicles exchanging data with each other and the infrastructure, has proven to improve traffic safety and increase the efficiency of transportation systems. DSRC (Direct Short Range Communication), which is based on IEEE $802.11 \mathrm{p}$, has been the subject of extensive standardization, product development and field trials by all stakeholders, proving its benefit for V2X. Recently, we assisted to a wide spread of COTS (Commercial Off The Shelf) solutions for V2X in the market. STM launched a cooperation with Autotalks for the co-development of a mass-market optimized V2X chipset [4]. CRATON2, designed for autonomous vehicles, integrates a mobility optimized IEEE802.11p modem, and supports IEEE $802.11 \mathrm{a} / \mathrm{b} / \mathrm{g} / \mathrm{n} / \mathrm{ac}$ to enable external WiFi for supplementary value-added services [4].
LinkBird-MX is a part of the development kit NEC C2XSDK, supporting fast application development in the field of ITS [5]. Many other companies have been released other several V2X devices, not reported here for sake of space. Since both hardware and software are available as COTS devices, the purpose of this paper is to develop a simulating model able to provide estimation of the link performance and to quantify the robustness of such wireless communication systems. This work is focusing on V2X physical layer based on IEEE $802.11 \mathrm{p}$, and designing the simulation tool in Matlab/Simulink environment.

At the state of the art, numerous studies have been carried out in literature and different models of 801.11p PHY Layer have been proposed, e.g. [6-8]. For example, the authors in [8] used a vehicular non-stationary channel model to implement the IEEE 802.11p PHY layer. However, all these developed models lack of considering the hardware part of real devices, such as transmitted power, transmitter and receiver antenna gains, receiver noise figure, receiver sensitivity, transmitter and receiver oscillator phase noise, implementation loss factors of the digital base band. Moreover, state of art PHY Layer models often lack of considering some impairments related to the propagation environment, dependent on operating scenario (e.g. urban, highway, suburban,..) and vehicle conditions (distance, speed, direction). Some works, e.g. [9], in literature consider the impact on link performance of hardware impairments, such as receiver noise figure non linearity, but adopting very simple freespace line-of-sight (LOS) channel models, not useful for real ITS scenarios. In [10] a complex traffic model is considered, but mainly operating at a higher abstraction level, without any link to the PHY layer, or hardware or channel constraints. Other models, e.g. [11], have been proposed considering the impact on communication performance at PHY Layer of complex channel models and even hardware non-idealities. However, the focus of [11] is limited to indoor applications, operating at mmwaves on short distance of tens of meters, considering static or slow moving nodes, which is suitable for industry 4.0 scenarios, not for ITS and autonomous vehicles.

To solve the above issues, this work shows a new Matlab/ Simulink model of V2X transceiver, implementing the PHY Layer of IEEE 802.11p, which takes also into account the transmitter and receiver device specifications (power out, antenna gain, noise, etc.) and the environment parameters (scenarios, speed vehicles, distance, etc.). As showed in Fig. 1, thanks to this model it will be possible to forecast the performance in terms of bit error rate (BER), receiver signal strength indicator (RSSI), packet delivery ratio (PDR), i.e. ratio between successfully received and transmitted packets, and so on. On the other hand, it is also possible to set as input some V2X target performance to generate device specifications useful 
during the system design process. The structure of the paper is as follows. Section 2 deals with the IEEE 802.11p PHY Layer state of art. The implementation of the model and the transceiver architecture are shown in Section 3. Validation process and simulation results are presented in Section 4. Conclusions are drawn in Section 5.

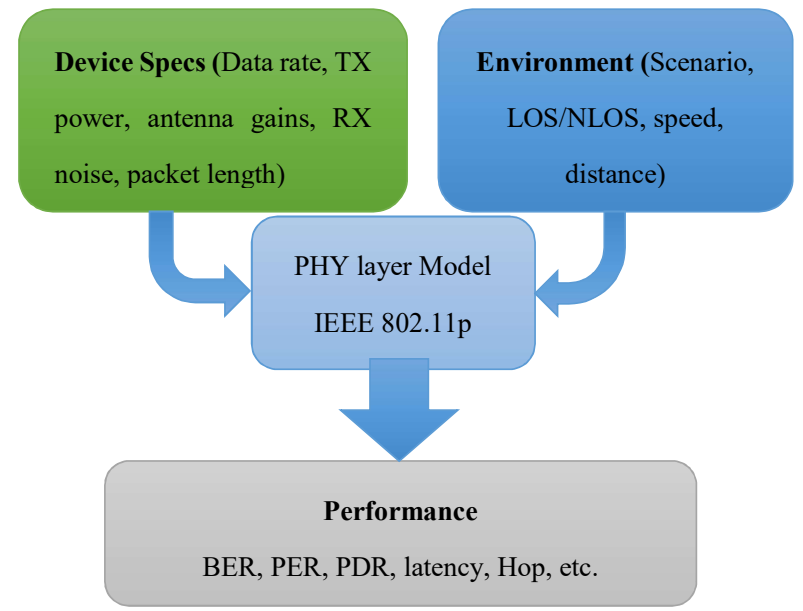

Fig. 1: Example use case of the proposed PHY Layer model

\section{IEEE 802.11p PHY Layer}

IEEE $802.11 \mathrm{p}$ is an approved amendment to the IEEE 802.11 standard to add wireless access in vehicular environments (WAVE) [12]. Dedicated applications include toll collection, red light duration broadcast at traffic lights or hot spots for transferring maps, routing information or traffic jams. But also, active accident warnings should be transferred from cars in a traffic jam to the oncoming cars. The 802.11p 5.9 GHz band uses a 52subcarrier orthogonal frequency division multiplexing (OFDM) scheme with a data rate between 3 and $27 \mathrm{Mbit} / \mathrm{s}$, depending on the adopted modulation scheme. OFDM is a multicarrier modulation technique, which divides the transmitted bit stream into many different sub-stream and send them over many different subcarriers [12]. The 52 subcarriers are composed by 48 data subcarriers and 4 pilot subcarriers. The center zero subcarrier is not used ("DC" or "Null"). The possible data subcarrier modulation formats are BPSK, QPSK, 16QAM, 64QAM with coding rate of $1 / 2,2 / 3$ or $3 / 4$. Pilot subcarriers are modulated using BPSK with a known magnitude and phase. Each OFDM subcarrier carries a single modulated data symbol, or "constellation point", along with its magnitude and phase information. One or more concatenated OFDM symbols form an OFDM burst. The frame structure of an $802.11 \mathrm{p}$ burst contains a preamble field followed by a SIGNAL field and multiple data fields. At the start of the burst, a preamble is transmitted at a known magnitude and phase. The preamble is used for synchronization and channel equalization. The SIGNAL field is transmitted using BPSK, and contains the length, modulation type, and data rate information. To complete the burst, multiple OFDM symbols containing the data bits are appended

\section{Implementation of the Model}

The current research aims at developing a transceiver model for $\mathrm{V} 2 \mathrm{X}$ communication to evaluate the reliability of IEEE $802.11 \mathrm{p}$ protocol. Many aspects affect the reliability of any wireless link, such as the multipath propagation and delay involved in processing critical messages. The distance between the receiver and the transmitter affects the reliability of the wireless connection, because when the distance increases the pathloss increases causing high error rate. So that, the modelling process for PHY layer, starts identifying the system components. We distinguish between the digital and the analog parts in both transmitter and receiver. An important component is represented by the radio channel, which mainly affects the reliability of the communication link. The entire model, developed in Matlab/Simulink, is valid taking into account those assumptions:

- Baseband-equivalent Matlab/Simulink model

- V2X communication between 2 nodes/vehicles

- The two nodes/vehicles are able to communicate with each other through a single hop (multi-hop extension is addressed in Section 4)

- $\quad$ Relative speed between the moving nodes is used

- MAC and higher levels of the ISO/OSI stack are not included in the model, whose outputs, however, can be used as inputs for higher level modelling/simulation tools.

The transmitter is the one who generates the signal to be sent over the channel. Before sending it, the digital part is in charge to process the signal using a specific adaptive modulation and coding scheme according to the standard protocol. To begin the OFDM signal creation process, the input data bit stream is encoded with convolutional coding and Interleaving. Each data stream is divided into groups of "n" bits (1 bit -BPSK, 2 bits -QPSK, 4 bits -16QAM, or 6 bits -64QAM) and converted into complex numbers $(\mathrm{I}+\mathrm{jQ})$ representing the mapped constellation point. The modulation scheme is selected according to the Signal to Noise Ratio (SNR) estimate at the receiver, since the model uses an Adaptive Modulation and Coding (AMC) approach. In this way, it is possible to adapt the bit rate according to the link quality. Then 52 bins of the IFFT block are loaded. 48 bins contain the constellation points, which are mapped into frequency offset indexes ranging from -26 to +26 , skipping the 4 Pilot and zero bins. At this point, the IFFT is computed giving a set of complex timedomain samples representing the combined OFDM subcarrier waveform. To complete the OFDM symbol, a $1.6 \mu$ s duration Cyclic Prefix is then added (as guard interval) to the beginning of the OFDM waveform. This produces a "single" OFDM symbol with a time duration of $8 \mu \mathrm{s}$ in length. The process is repeated to create additional OFDM symbols for the remaining input data bits. To complete the OFDM frame structure, the single OFDM symbols are concatenated together, and then appended to a $32 \mu$ s Preamble and an $8 \mu$ s SIGNAL symbol. This completes the OFDM frame and is ready to be transmitted as an OFDM Burst. The digital part of the receiver basically performs the reverse operation as the transmitter. Firstly, the CP is removed, and the received signal is converted to the frequency domain using, in this case, the FFT algorithm. Thus, a process to separate all these subcarriers is needed. First, the guard bands are removed, and then, a disassembling is performed to obtain pilots, data, and preamble. The preamble is used in the channel estimator, which calculates channel coefficients to be used 
in the demapper to perform an equalization of the data, and so, compensate the frequency-selective fading of the multipath propagation channel. Once the data has been demapped, it enters the Viterbi decoder block. The analog part has been modelled starting from the link budget analysis, which accounts the specification of the hardware part of the device [13]. The receiver power in a 802.11 link is determined by these factors: antenna gains; transmitted radiation power; attenuation factors. A link is possible only if the power at the receiver is greater than the receiver sensitivity: the difference between the received signal level and the receiver sensitivity is called link margin. This analysis is calculated by Eq. 1 where: $\mathrm{P}_{-} \mathrm{RX}$ is the receiver power; $P_{-}$TX is the transmitter power; G TX is the transmitter antenna gain; L_RX are losses due to cable, connectors etc.; L_PL is the path loss; L_M are miscellaneous losses due to fade margin, polarization misalignment etc.; G_RX is the antenna gain of receiver; L_RX are losses from receiver.

$P_{R X(d B m)}=P_{T X(d B m)}+G_{T X(d B i)}-L_{T X(d B)}-L_{P L(d B)}-$

$L_{M(d B)}+G_{R x(d b i)}-L_{R X(d B)}$

To obtain a measure of the transmission performance we must necessarily model the noise power and get a realistic SNR. The noise factors are represented by: devices thermal noise, noise figure, phase noise and multipath fading. The thermal noise is given by Eq. 2 where: $\mathrm{k}$ is the Boltzmann's constant, $\mathrm{T}_{-} 0$ is the temperature in kelvin, $\mathrm{B}$ w is the signal bandwidth, $\mathrm{F}$ is the noise figure. Although signal is transmitted in baseband, the model considers the phase noise caused by the phase fluctuating of the oscillator. The phase noise is defined by: phase noise level $(\mathrm{dBc} / \mathrm{Hz})$, frequency offset $(\mathrm{Hz})$ and sample rate $(\mathrm{Hz})$. For the SNR statistics we main refer to the Eq. 3 and Eq. 4 where R_b is the bit rate and $\mathrm{Eb} / \mathrm{N}_{0}$ is a normalized SNR, also known as the "SNR per bit".

$P_{N}=k T_{0} \cdot B_{w} \cdot F$

$\frac{S}{N}=\frac{P_{T X} \cdot G_{T X} \cdot G_{T X}}{k T_{0} \cdot B_{W} \cdot F \cdot L_{T X} \cdot L_{T X} L_{P L} \cdot L_{M}}$

$\frac{S}{N}=\left(\frac{E_{b}}{N_{0}}\right) \cdot\left(\frac{R_{b}}{B_{w}}\right)$

The term channel refers to the medium between the transmitting antenna and the receiving antenna. Mathematically, the radio propagation channel can be represented by a time-variant impulse response $\mathrm{h}(\mathrm{t}, \tau)$ and the received signal $\mathrm{y}(\mathrm{t})$ can be obtained by convolving the transmit signal $\mathrm{x}(\mathrm{t})$ with the time-variant impulse response $h(t, \tau)$. The three key components of the channel impulse response are path loss, shadowing, and multipath fading [14]. Path loss is the attenuation in power density of an electromagnetic wave as it propagates through space. It is expressed in $\mathrm{dB}$ by the Eq. 5 where: $\mathrm{n}$ is the path loss exponent; $d$ is the distance between the transmitter and the receiver; $\mathrm{d} 0$ is a reference distance. Different propagation environments are simulated such as rural, urban, sub-urban and highway. The PL parameters for LOS condition are reported in Table $1[15,16]$. To take into account the NLOS case, we consider an attenuation factor from $10 \mathrm{~dB}$ to $25 \mathrm{~dB}$ in received power [17]. Shadowing is the effect that the received signal power fluctuates due to objects obstructing the propagation and is represented with the random process X_ $\sigma$, having a Gaussian distribution with zero mean value and standard deviation $\sigma$ in Table 1 .

\begin{tabular}{|c|c|c|c|}
\hline Environment & $P L\left(d_{0}\right)(d B)$ & $\mathbf{n}$ & $\sigma(d B)$ \\
\hline Rural & -61.1 & -1.79 & 3.3 \\
\hline Highway & -59.7 & -1.85 & 3.2 \\
\hline Urban & -68.5 & -1.61 & 3.4 \\
\hline Suburban & -65.0 & -1.57 & 4.2 \\
\hline
\end{tabular}

Multipath represents the multiple reflected copies of the transmitted signal that arrive at the receiver at different time instants $\left(\tau \_n\right)$ and at different power levels (a_n). This characteristic of the multipath phenomena is described by Power Delay Profile (PDP). Such power-delay-profile models can be simulated using discrete-time Tapped Delay Line (TDL) filter with $\mathrm{N}$ number of taps with variable tap gains. Taps of delay are usually specified in standards like 802.11-14/0259r0 [18], an amendment to the 802.11 standard, which specifies $802.11 \mathrm{p} \mathrm{V} 2 \mathrm{~V}$ radio channel models. It defines the TDL models for five scenarios: Rural LOS; Urban approaching LOS; Street Crossing NLOS; Highway LOS; Highway NLOS. To render our model time variant, we must add some randomness. Rician fading distribution is applied in the case that a LOS condition exists between the transmitter and the receiver. Rayleigh distributions are defined for fading of a channel when all the received signals are reflected signals and there is no dominant component. Fig. 2 shows the complete model for the IEEE 802.11p transceiver. Each block has been named intuitively with proper label, and additional block are added for statistics (bit error rate, packet error rate, constellation, SNR etc.).

\section{Validation and Simulation Results}

The verification procedures are configured by running the system model through an Additive White Gaussian Noise (AWGN) channel. The simulated BER performance for uncoded transmission with BPSK, QPSK, 16QAM and 64 QAM are compared with theoretical approximations (dashed lines), see Fig. 3(a). The error probability for signals like BPSK, QPSK can be found in any digital communication textbook, [19]. The simulation curves are obtained removing the convolutional coding and the decoder block from the Simulink model thus neglecting the analog part and the impairments. We can conclude that the overall model performance with uncoded transmission is verified, since it matches the theoretical expectations. In Fig. 3(b) we compare BER performance of coded transmission over AWGN channel for all available combinations of modulation schemes and coding rates. The BER vs. SNR performance of 16 and 64 QAM is poorer than that of BPSK and QPSK. This is mainly because for a given SNR value, the symbols of 16 and 64 QAM constellations have to be more densely spaced than the symbols of BPSK and QPSK constellations, which implies higher error probabilities. As next verification step, the PHY layer model simulation results have been compared to measurements in real scenarios, considering the same environments and device parameters. 


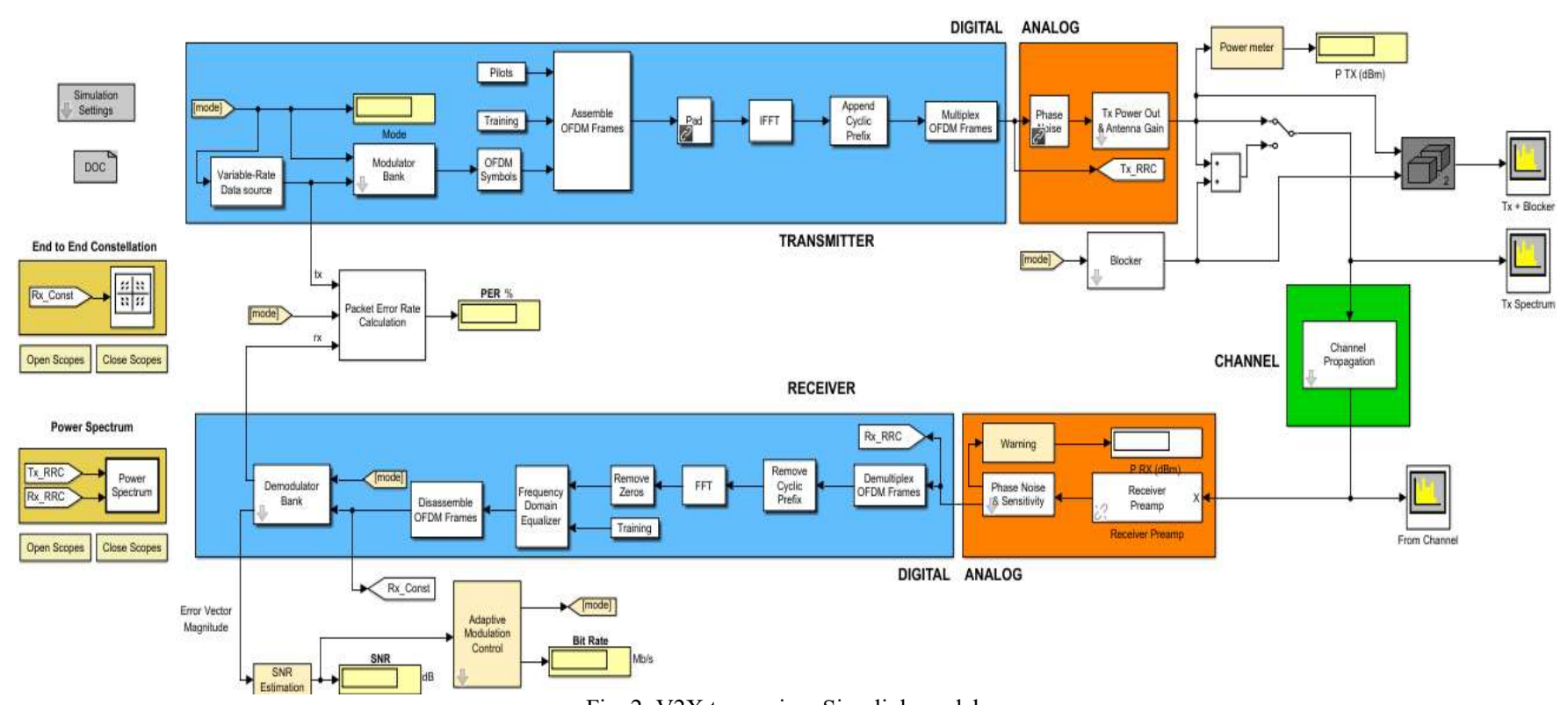

Fig. 2: V2X transceiver Simulink model

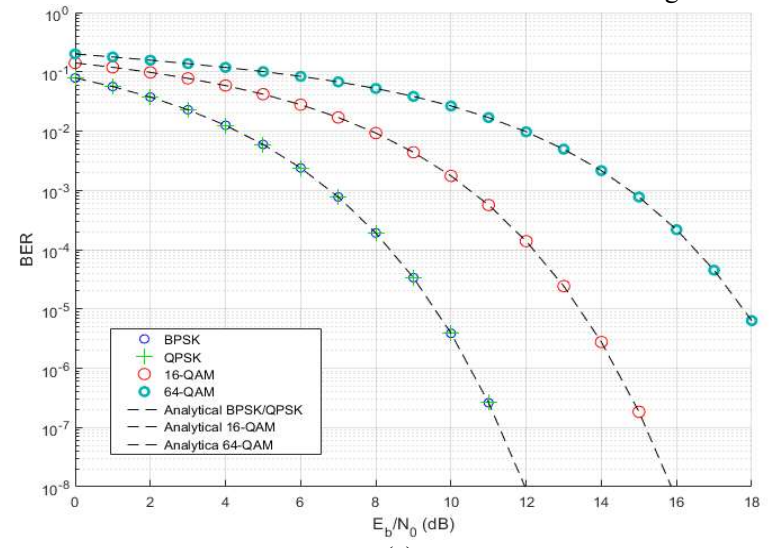

(a)

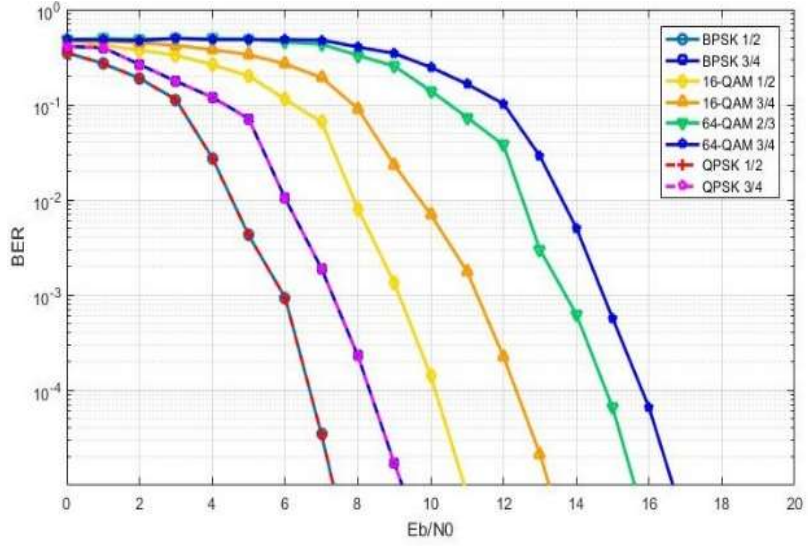

(b)

Fig. 3: BER vs. Eb/ $/ \mathrm{N}_{0}$ for uncoded(a) and coded (b) transmission. Data in (a) are compared to analytical results from theory

Measurements were performed in, or near, Pittsburgh PA, USA using two cars equipped with a pair of NEC LinkBird-MX [5]. The devices were set to transmit with QPSK $1 / 2$ modulations at $6 \mathrm{Mbps}$ data rate, to get the largest possible communication range. For the settings parameters we refer to Table 1 on [20]: center frequency $5.9 \mathrm{GHz}$, channel bandwidth $10 \mathrm{MHz}$, data-rate $6 \mathrm{Mbps}$, measured transmitted power $10 \mathrm{dBm}$, antenna gain $5 \mathrm{dBi}$. The goal of the measurements was to study the PDR as function of distance separately for each on-the-road scenario (urban, suburban, highway) in both LOS and NLOS conditions, see Fig. 4. In Fig. 4 the solid lines without markers indicate the mean of the measurements taken in the real world, while dashed lines indicate the $20 \%$ and $80 \%$ quantiles. The simulation results are indicated instead, with the solid lines with the circle markers. In Fig. 4(a-c) simulation results are always close together with the mean of the real measurements, except for the highway environment in NLOS condition. Fig. 4(d) shows the reliable connection range under different LOS conditions. For reliable connection we intend the maximum range in which the PDR is close to $90 \%$. Our simulation results are slightly worse with respect to the real measurements in LOS condition getting a worsening around $10-15 \mathrm{~m}$ for all three environments at $90 \%$ of PDR. In NLOS simulations, the results are slightly better than the mean of the real data.
The estimation results that are obtained with the proposed model have ben also compared with the results achievable in a real world measurement campaign, in Tuscany [21], using the NEC LinkBird-MX with omni-directional, 108 $\mathrm{mm}$ long WiMo antenna with $5 \mathrm{dBi}$ gain. Also in this case we achieved a good agreement between experimental measurements and predicted model results.

The mobility is an important V2X communication factor. This section examines the model dependence vs. speed in urban and highway environments. We decided to keep the same settings parameters of the NEC LinkBird. In this way, it is possible to use the model to know what performance comes out for a given devices specification. Figs. 5(a-b) show the PDR vs. speed (0-220 Km/h) in LOS and NLOS conditions at $200 \mathrm{~m}$ for all possible data rates. As expected, BPSK $1 / 2$ and QPSK $1 / 2$ have always the best performance in all scenarios. Fig. 5(a) shows that BPSK $1 / 2$ has a PDR from $90 \%$ to $20 \%$ as the speed increases from 0 to $220 \mathrm{~km} / \mathrm{h}$. In NLOS case, the PDR drops at $18 \%$ at zero speed for BPSK $1 / 2$. These results show the communication getting worse in presence of obstruction, especially at high speed. For highway scenarios it was used only the most robust modulation (BPSK 1/2) plotting different curves at different speed, to get information about the coverage in $1000 \mathrm{~m}$, see Figs. 5(c),(d). The NLOS case shows how the obstruction has 


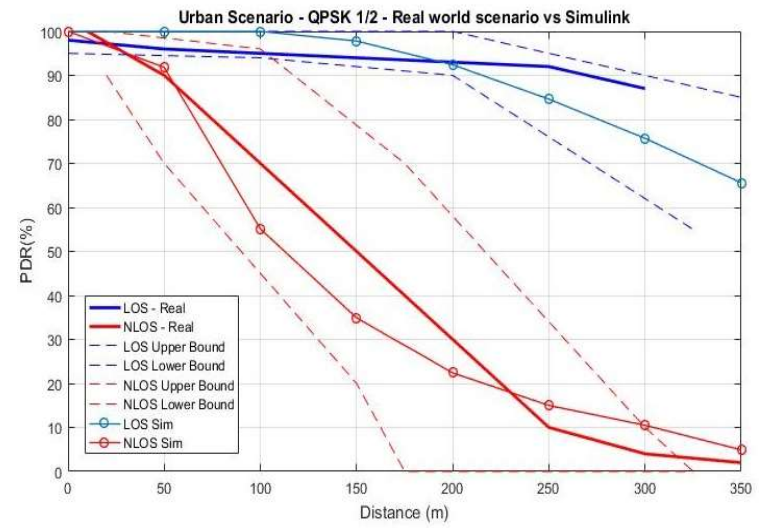

(a)

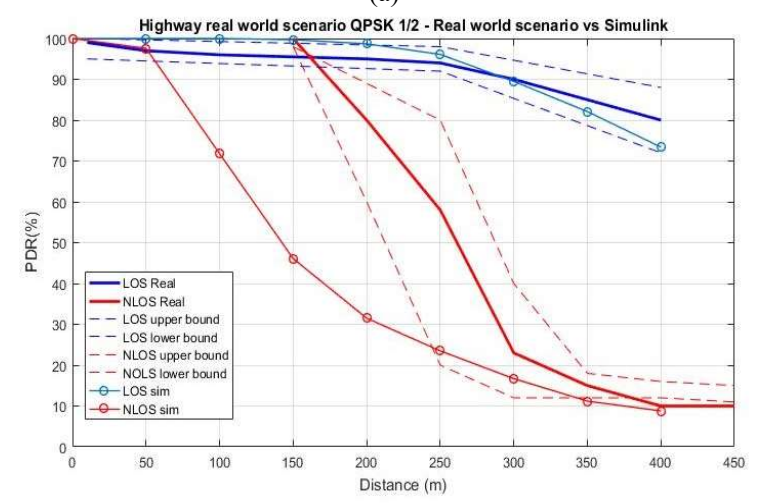

(c)

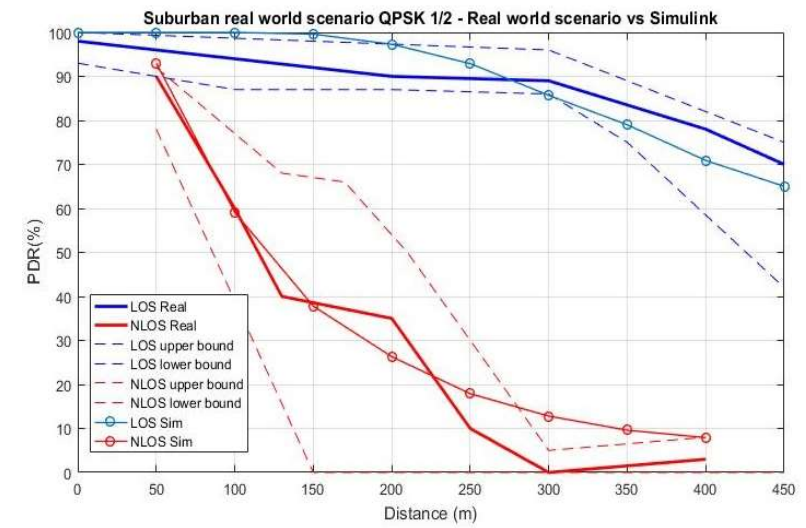

(b)



(d)

Fig. 4: PDR vs. distance for real measurements and simulation in different scenarios: (a) Urban; (b) Suburban; (c) Highway

a drastic negative impact over all statistics. Those graphs are depicted just as a specific application case of such transceiver model. It is obvious that better performance could be obtained in the same propagation environment using better specifications, and so, setting differently the model parameters. Starting from these observations, it is possible to show how the multi-hop communication scheme enhances overall performance, studying the RSU placement problem in a vehicular network. In this problem, each vehicle can access RSUs in two ways: I) direct delivery, which occurs when the vehicle enters the transmission of each RSU, and II) multi-hop relaying, which takes place when the vehicle is out of RSU's transmission range. In this section we assume that the target is to cover a distance of $1 \mathrm{Km}$ keeping a high PDR. Referring to Fig. 5(c), in a highway scenario and in static condition (zero speed) the PDR at $1000 \mathrm{~m}$ is $42.1 \%$. Without loss of generality, it is possible to split the full range in $\mathrm{N}$ segments ( $\mathrm{N}$ hops) placing one $\mathrm{RSU}$ in each and so increasing the efficiency of the transmission. This is possible if the probability of error from each segment is independent. Table 2 shows how the PDR increases in the static case, from $42.1 \%$ of a single hop to $70.05 \%$ for two hops of $500 \mathrm{~m}$. The best performance are obtained splitting the distance in four segments (quad-hops) reaching a $96.82 \%$ of PDR. Table 3 shows the performance obtained in case a moving car arriving with a speed of $120 \mathrm{Km} / \mathrm{h}$. Also in this case the goal is to forward packets in $1000 \mathrm{~m}$ maintaining high the PDR. The purple line of Fig. 5(c) shows that $1000 \mathrm{~m}$ are covered with a PDR of $30.7 \%$ in a single hop. In two hops the PDR increase at $62.38 \%$, in four at $85 \%$. Also in this case is possible to reach better performance using different configuration parameters.

\section{Conclusions}

This work is focused on developing and verifying a simulation model of the IEEE $802.11 \mathrm{p}$ transceiver. The validation was carried out comparing the analytic curves of BER vs. Eb/ $\mathrm{N}_{0}$ with respect to the simulation results, firstly in AWGN for uncoded transmission, then for coded transmission. Physical layer was evaluated using path loss, multipath and fading propagation, with multiple tests for urban, suburban, rural, highway scenarios. A comparison was made considering real word experimental data from measurements in different areas (urban, suburban, highway) and conditions (LOS, NLOS), using the hardware apparatus NEC LinkBird-MX. 16-QAM and 64QAM modulations show a low PDR in most of the cases, due to the high data rate, so are considered not reliable for safety applications such as autonomous driving. The impact of multi-hop vehicular network configuration has been also discussed and the proposed model can help facing the RSUs placement problem. More in general, thanks to the proposed model, it is possible to forecast the performance in terms of BER and PDR given the operating conditions/scenarios and the hardware configuration, or it is possible to set as input some V2X target performance to generate device specifications for the system design.

\section{References}

1. Standard specification for telecommunications and information exchange between roadside and vehicle systems $-5 \mathrm{GHz}$ band dedicated short range communications (DSRC) Medium Access Control (MAC) and Physical Layer (PHY) Specifications, ASTM E2213, Nov. 2006.

2. F. Pieri et al., "Consumer electronics is redesigning our cars? Challenges of integrated technologies for sensing, computing and storage", IEEE Consumer Electronics Magazine, September 2018 
3. E. Massaro, et al., "The Car as an Ambient Sensing Platform", Proceedings of the IEEE, vol. 105, n. 1, pp. 1-5, 2017

4. Auto-talks.https://www.auto-talks.com/product/craton2/.

5. Network Division NEC Laboratories: CAR-2-X Communication SDK. User Guide. NEC Europe Ltd., 29.10.2009 Version 1.5.2.

6. G. Kiokes, et al. "Simulation-based performance analysis and improvement of orthogonal frequency division multiplexing $-802.11 \mathrm{p}$ system for vehicular communications", IET Intelligent Transport Systems, 2009, pp $429-436$.

7. S. Eichler, "Performance evaluation of the IEEE 802.11p WAVE communication standard", IEEE VTC, pp. 2199 - 2203, 2007.

8. L. Bernado, N. Czink, Th. Zemen, P. Belanovie, "Physical layer simulation results for IEEE $802.11 \mathrm{p}$ using vehicular non - stationary channel model", IEEE Communications Workshop, 2010, pp $1-5$.

9. S. Saponara, et al., "System-level modelling/analysis and LNA design in low-cost automotive technology of a V2X wireless transceiver", IEEE RTSI 2017, pp. 1-5

10. Linguo Chai et al., "Basic simulation environment for highly customized connected and autonomous vehicle kinematic scenarios", Sensors, vol. 17, n. 9, 2017

11. S. Saponara, et al. "Exploiting mm-Wave Communications to Boost the Performance of Industrial Wireless Networks", IEEE Trans. Industrial Informatics, vol. 13, n. 3, pp. 1460-1470, 2017
12. $802.11 \mathrm{p} \mathrm{Standards.} \mathrm{http://standars.iee.org/getieee802/download/-}$ 802.11p-2010.pdf.

13. S.R. Saunders: Antennas and propagation for wireless communication systems, Wiley 1999.

14. Andrea Goldsmith, "Wireless Communications," Cambridge University Press, 2005,644 pp.

15. J. Kunisch, J. Pamp, "Wideband car-to-car radio channel measurements and model at $5.9 \mathrm{GHz}$," IEEE VTC, 2008. pp. 1 -5.

16. L. Cheng, et al., "Mobile vehicle-to-vehicle narrow-band channel measurement and characterization of the $5.9 \mathrm{GHz}$ dedicated short range communication (DSRC) frequency band," IEEE Journal on Selected Areas in Comm., vol. 25, n. 8, pp. 1501-1516, 2007.

17. Jhihoon Joo et al., "Measurement based V2V path loss analysis in urban NLOS scenarios", IEEE ICUFN 2016, pp. 73 - 75.

18. M. Kahn, IEEE 802.11 Regulatory SC DSRC Coexistence Tiger Team V2V Radio Channel Models, doc. IEEE 802.11-14/0259r0, 2014.

19. J. G. Proakis, Digital Communications . 3rd ed., 1995.

20. R. Meiereles et al., "Experimental study of the impact of vehicular obstructions in VANETs", IEEE Vehicular Net. Con., 2010, pp. 338-345 21. M. Renda et al.,"IEEE 802.11p VANets: experimental evaluation of packet inter-reception time", Computer Com., vol.75, pp.26-38, 2016

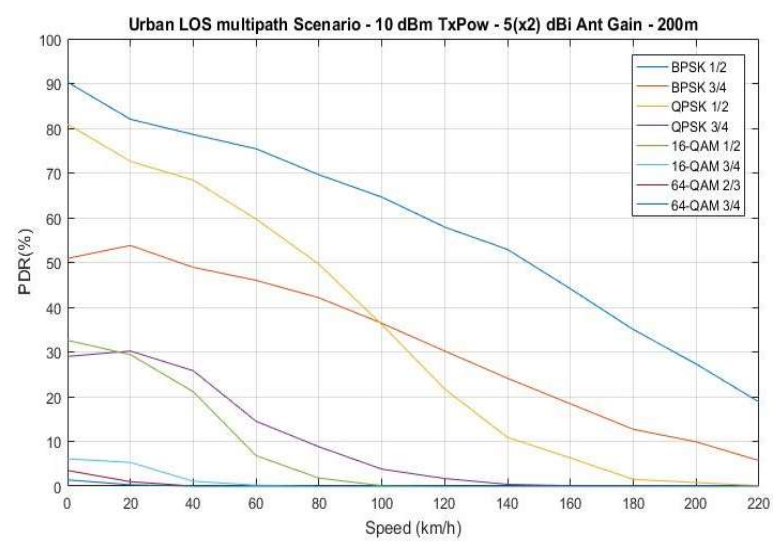

(a)

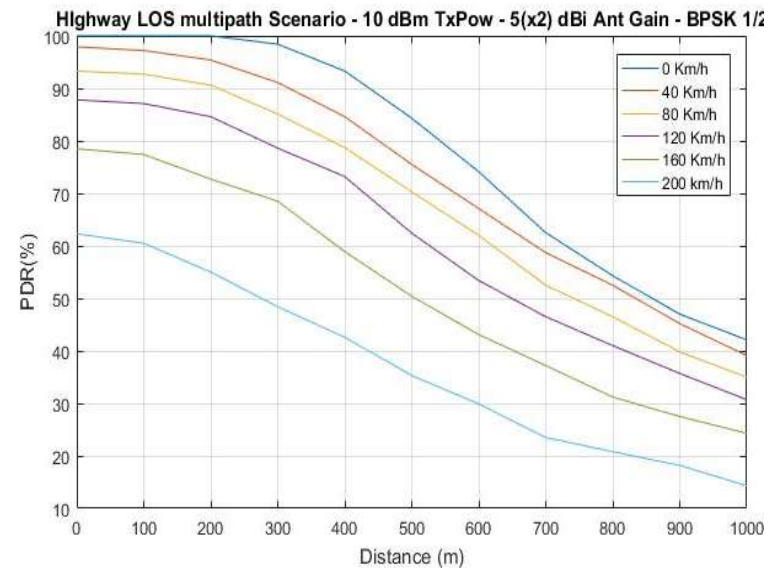

(c)

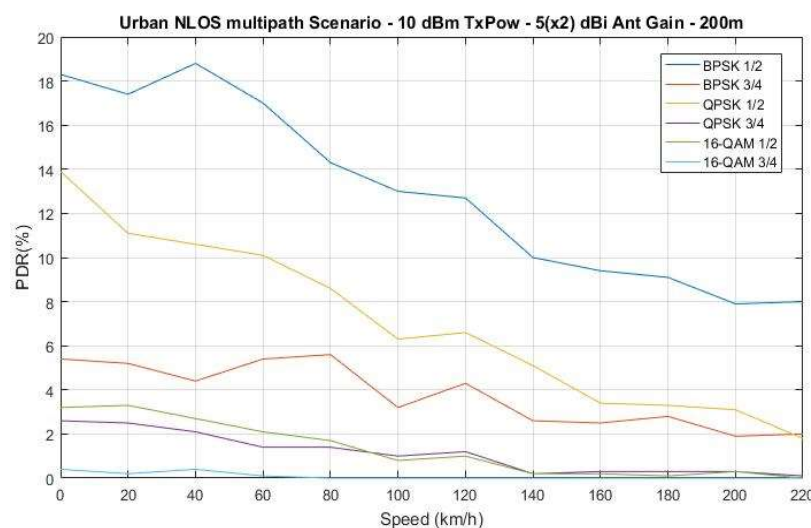

(b)

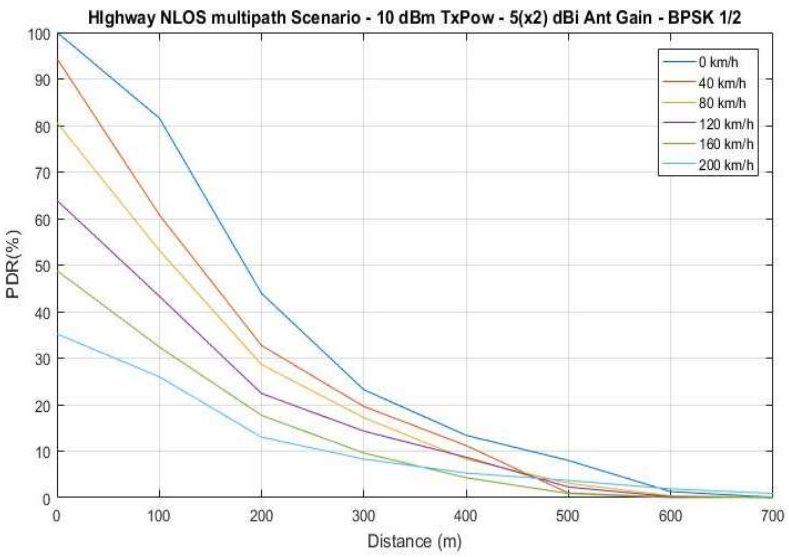

(d)

Fig. 5: PDR vs. speed at 200m: Urban LOS(a); Urban NLOS(b). PDR vs. distance: (c) Highway LOS; (d) Highway NLOS

Table 2. PDR in multi-hop communication

\begin{tabular}{ccc}
\hline \#hop & $P D R_{\#}(\%)$ & $P D R_{n}(\%)$ \\
\hline $1(1000 \mathrm{~m})$ & 42.1 & 42.1 \\
$2(500 \mathrm{~m})$ & 84.37 & 71.05 \\
$4(250 \mathrm{~m})$ & 99.2 & 96.82 \\
\hline
\end{tabular}

Table 3. PDR in multi-hop communication.

\begin{tabular}{ccc}
\hline \#hop & $P D R_{\#}(\%)$ & $P D R_{n}(\%)$ \\
\hline $1(1000 \mathrm{~m})$ & 30.7 & 30.7 \\
$2(500 \mathrm{~m})$ & 84.3 & 62.38 \\
$4(250 \mathrm{~m})$ & 99.2 & 85.00 \\
\hline \multicolumn{2}{c}{ Speed $120 \mathrm{~km} / \mathrm{h}-0 \mathrm{~m}-\mathrm{PDR}_{0}=87.8 \%$}
\end{tabular}

Speed $120 \mathrm{~km} / \mathrm{h}-0 \mathrm{~m}-\mathrm{PDR}_{0}=87.8 \%$

This work has been supported by PRA2017 (Pisa University) and EPI (European Processor Initiative) H2020 projects 